\title{
COVID-19 NO ESTADO DE MINAS GERAIS: ANÁLISE ESTATÍSTICA DO NÚMERO DE ÓBITOS RELACIONADO À POPULAÇÃO ESTIMADA DOS MUNICÍPIOS MINEIROS UTILIZANDO O SOFTWARE MAPLE®
}

\author{
Ellen Werneck Resende - ellen.resende@alunos.unis.edu.br \\ Loaisy Machado Rodrigues - loaisy.rodrigues@alunos.unis.edu.br \\ LuanyDinalva Tavares - luany.tavares@alunos.unis.edu.br \\ Tiago BittencourtNazaré - tiago@unis.edu.br
}

RESUMO:O COVID-19 trata-se de uma doença infecciosa causada pelo coronavírus. Esse resultou em uma pandemia que se espalhou por cidades do mundo inteiro, infectando suas populações e, em muitos casos, levando a óbito. Nesta perspectiva, o objetivo do presente trabalho foi realizar um estudo analisando o cenário da COVID-19 no estado de Minas Gerais antes de iniciar a vacinação, através de dados extraídos diretamente da Secretaria de Estado de Saúde de Minas Gerais e do Instituto Brasileiro de Geografia e Estatística (IBGE). Além disso, para desenvolver o trabalho fez-se necessário utilizar asferramentasMicrosoft Excel 2016 e Maple 17 com o intuito de organizar os dados, realizar cálculos e análises estatísticas da amostra, como, por exemplo: média, desvio padrão, variância e curtose. Através dos cálculos citados foi possível gerar gráfico e visualizar o cenário das cidades mineiras frente à pandemia.Ao final da análise dos resultados, observou-se que a cidade que obteveo pior cenário frente à pandemia foi Aracitaba, com 2.059 habitantes e 7 óbitos.Já a cidade que obteve o melhor cenário foiBerilo,com 11.872 habitantes e apenas 0 óbitos. Mas, é importante ressaltar que através dos cálculos estatísticos evidenciou-se que as cidades mineiras tiveram comportamentos bem semelhantes, pois não houve muita variação nos resultados obtidos.

Palavras-Chave: Coronavírus.Óbitos. Estatística.Minas Gerais.

ABSTRACT:COVID-19 is an infectious disease caused by the coronavirus. This resulted in a pandemic that spread to cities around the world, infecting their populations and, in many cases, leading to death. In this perspective, the objective of the present work was to carry out a study analyzing the current scenario of COVID-19 
in the state of Minas Gerais, using data extracted directly from the State Department of Health of Minas Gerais and the Brazilian Institute of Geography and Statistics (IBGE). In addition, to develop the work it was necessary to use the tools Microsoft Excel 2016 and Maple 17 to organize the data, perform calculations and statistical analysis of the sample, such as: mean, standard deviation, variance and kurtosis. Through the aforementioned calculations, it was possible to generate a graph and visualize the scenario of mining towns facing the pandemic. At the end of the analysis of the results, it was observed that the city that had the worst scenario in the face of the pandemic was Aracitaba, with 2,059 inhabitants and 7 deaths. The city that obtained the best scenario was Berilo, with 11,872 inhabitants and only 0 deaths. However, it is important to note that through the statistical calculations it became evident that the cities in Minas Gerais had very similar behaviors, as there was not much variation in the results obtained.

Keywords:Coronavirus.Deaths. Statistic. Minas Gerais.

\section{INTRODUÇÃO}

Ao final de 2019, na cidade de Wuhan, China, confirmou-se o primeiro caso de um vírus denominado coronavírus que ocasionou em uma pandemia que se estende até os dias de hoje. Segundo o aplicativo Coronavírus SUS 2.0.9 (2021), a doença COVID19 tem como sintomas: febre, tosse seca, cansaço, dor de cabeça, dor de garganta, perda de paladar ou olfato, dores e desconfortos no corpo, dificuldade de respirar e entre outros. Essa é uma doença causada pelo coronavírus e apresenta alto índice de contágio. Além disso, apresenta um espectro clínico variando de infecções assintomáticas a quadros graves que podem levar o indivíduo a óbito.

Segundo dados do painel Coronavírus Brasil 2.0 (2021), atualmente, o Brasil apresenta cerca de 15.812 .055 casos confirmados e 441.691 óbitos confirmados pela COVID-19, sendo 1.483.200 casos confirmados e 37.927 óbitos confirmados apenas no estado de Minas Gerais, região Sudeste do país.

Neste sentido, o presente artigo tem como objetivo analisar o cenário da COVID-19 em Minas Gerais antes de iniciar a vacinação, ou seja, desde o início da pandemia no Brasil, mês de março de 2020, até o dia 17/01/2021, data em que se iniciou a vacinação.

O estudo será desenvolvido através das ferramentasMicrosoft Excel 2016 e Maple 17, onde dados extraídos do site da Secretaria de Estado de Saúde de Minas 
Gerais e IBGEserão organizados e, posteriormente, trabalhados. Através das análises e dos cálculos realizadosrelacionando o número de óbitos de cada cidade e sua população estimada, será possível identificar qual foi o cenário dos municípios mineiros frente à pandemia da doença COVID-19.

\section{REFERENCIAL TEÓRICO}

\subsection{Microsoft Excel}

Segundo a Microsoft® (2021) o Excel trata-se de uma ferramenta capaz de organizar dados, gerar gráficos e tabelas de maneira atrativa com o intuito de facilitar a compreensão das informações.

\subsection{Maple 17}

Maple é um software matemático que combina o motor matemático mais poderoso do mundo com uma interface que o torna extremamente fácil de analisar, explorar, visualizar e resolver problemas matemáticos (MAPLESOFT $\left.{ }^{\circledR}, 2021\right)$. Ainda segundo o fabricante, esse software é capaz de resolver problemas matemáticos com grande facilidade, rapidez e precisão. Além disso, através de uma linguagem de programação sofisticada projetada especificamente para a matemática, desenvolve soluções complexas.

\subsection{Funções da Estatística}

Média é a soma de dois ou mais valores numéricos divididos pela contagem dos valores numéricos (MCFEDRIES, 2020). O autor ainda afirma que o termo média se refere à medida média mais comum, que neste sentido, trata-se de um valor médio.

De acordo com McFedries (2020), o desvio-padrão informa a variação dos valores em uma coleção segundo a média. Ainda segundo o autor mencionado, o desvio-padrão refere-se à uma medida da dispersão relativa dos dados da amostra. Fávero e Belfiore (2017) afirmam que o desvio-padrão é a raiz quadrada da variância.

A variância de um conjunto é uma medida de dispersão dos dados. Ela é o quadrado do desvio-padrão (MCFEDRIES, 2020).

Já a curtose, segundo McFedries (2020), refere-se à uma medida das causas em uma distribuição de valores.A curtose pode ser definida como o grau de achatamento de uma distribuição de frequências (altura do pico da curva) em relação a uma distribuição teórica (FÁVERO E BELFIORE, 2017). 


\section{METODOLOGIA}

Para o desenvolvimento do presente trabalho foi necessário extrair a base de dados de distribuição dos casos de COVID-19 no estado de Minas Gerais. A base encontra-se no site da Secretaria de Estado de Saúde de Minas Gerais, através do link http://coronavirus.saude.mg.gov.br/painel. Para download do arquivo em formato .xlsx deve-se acessar a aba Transparência. Posteriormente, deve-se selecionar as opções Portal da Transparência COVID-19 e Dados epidemiológicos, consecutivamente. Por fim, deve-se clicar na opção Painel de Monitoramento de Casos e realizar o download do arquivo nomeado como.XLSX Painel, conforme figura 1.

Figura 1. Acesso à base de dados de distribuição dos casos de covid-19 no estado de Minas Gerais

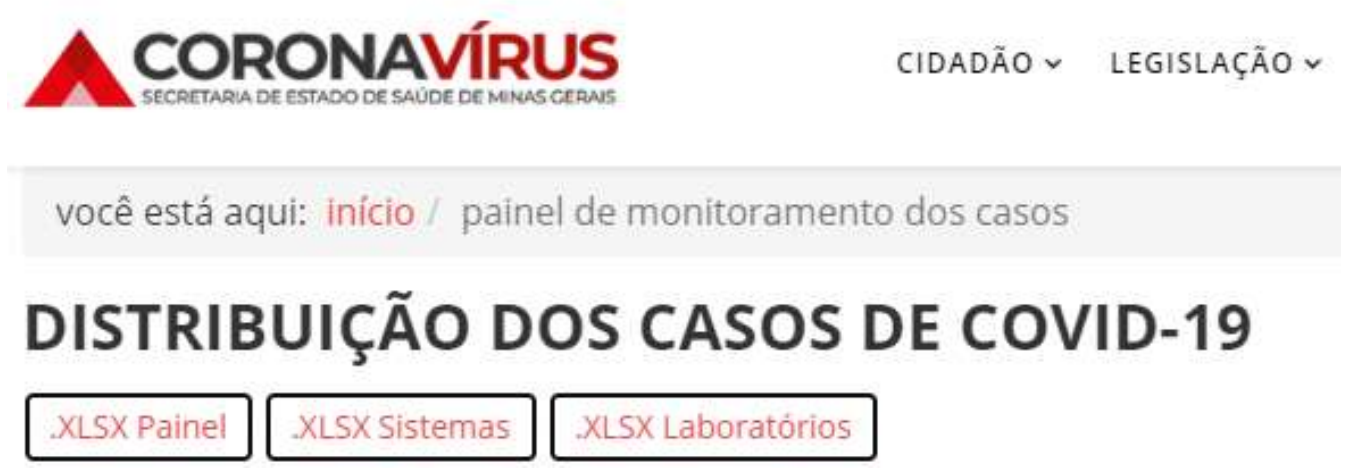

Fonte: Secretaria de Estado de Saúde de Minas Gerais (2021)

A base de dados acima mencionada foi extraída no dia 11/03/2021 e traz as seguintes informações: casos confirmados, recuperados, internados e óbitospela doença desde o dia 19/03/2020 em todas as 853 cidades do estado de Minas Gerais. Além disso, traz a data em que ocorreu cada óbito.

Além disso, fez-se necessário identificar a população estimada dos municípios do estado de Minas Gerais. Essa informação foi extraída diretamente do site do IBGE no dia 11/03/2021, através do link <https://www.ibge.gov.br/cidades-eestados/mg.html $>$ e conforme figura 2. 
Figura 2. Acesso à base de dados de população estimada dos municípios do estado de Minas Gerais

Cidades e Estados

Selecione um nível geográfico

Q Saiba mais no portal cidades@

Minas Gerais cỏdigo: 31
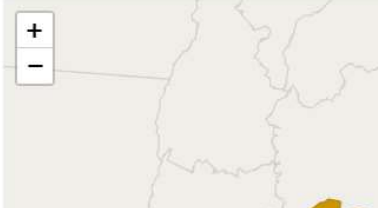

Área Territoria

População estimada

Densidade demográfica

Exportar -

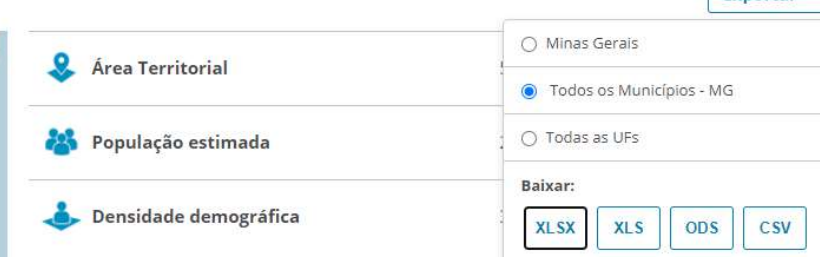

Fonte: IBGE (2021)

Por meio do link indicado, basta clicar na aba Exportar. Em seguida, selecionar a opção Todos os Municípios - MG e, por fim, baixar o arquivo em formato xlsx. Neste, é possível identificar diversas informações das cidades mineiras, tais como: área territorial, densidade, mortalidade, PIB per capita, o número de população e entre outras.

Para este estudo, utilizou-se dessa base apenas o número populacional das cidades mineiras, enquanto da base de dados de distribuição dos casos de COVID-19 utilizou-se apenas o número de óbitos.

Para a organização dos dados, utilizou-se a ferramenta Microsoft Excel 2016. Organizou-se em uma única planilha a lista dos munícipios mineiros e seus respectivos dados de óbitos e população estimada, distribuídos em três colunas. Para facilitar a visualização dos dados, inseriu-se uma Tabela Dinâmica. Após essa etapa, dividiu-se o número de óbitos pelo número da população estimada de cada cidade com o intuito de identificar qual foi o percentual de óbitos em relação a população dos munícipios.

Para análise dos valores obtidos, utilizou-se o software Maple 17, no qual calculou-se a média, a variância, o desvio-padrão e a curtose dos dados. As funções utilizadas no software acima mencionado foram: $m u:=\operatorname{Mean}(L)$, para identificar a média e sigma $:=$ StandardDeviation $(L)$ para encontrar o valor do desvio-padrão. Já a função responsável pelo cálculo da variância utilizada no presente trabalho foi Variance $(L)$, enquanto o cálculo da curtose foi feito utilizando a função Kurtosis(Normal(mu, sigma)). 


\section{RESULTADOS E DISCUSSÕES}

O estado de Minas Gerais tem 853 municípios e, segundo o IBGE (2020), uma população total de 21.292.666 habitantes. No período de estudo, 19/03/2020 a 17/01/2021, a cidade que obteve o maior número de óbitos foi a capital do estado, denominada Belo Horizonte, totalizando 2.855 óbitos.Do total de municípios mineiros, 68 obteve 0 óbitos, ou seja, 7,97\% dos municípios. Enquanto 93 cidades obtiveram apenas 1 morte, ou seja, 10,90\% dos municípios.

Considerando apenas o número de óbitos e desconsiderando o número populacional, a cidade que esteve no pior cenário em relação à pandemia daCOVID-19 foi a capital mineira Belo Horizonte, porém essa tem uma população estimada de 2.521.564 habitantesdestacando-se por sera cidade com maior número populacionaldo estado, conforme gráfico 1.

Gráfico1. População x Óbitos

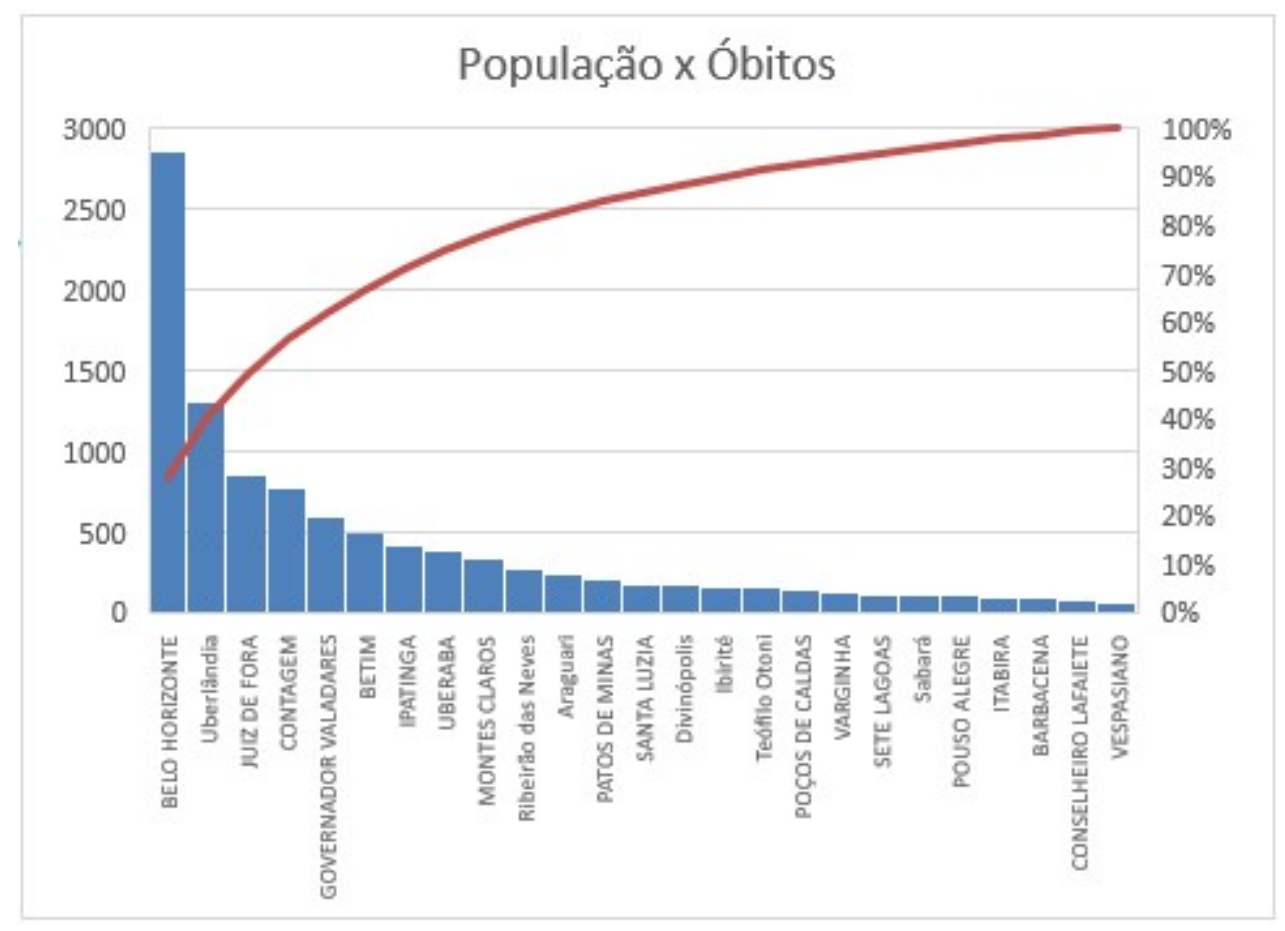

Fonte: Autores (2021)

Dessa forma, a probabilidade da cidade de Belo Horizonte ter o maior número de óbitos é muito alta, ou seja, os seus dados não são proporcionais com os das demais cidades.

Neste sentido, realizou-se uma análise relacionando o número de óbitos com o número de população estimada de cada cidade, com o intuito de identificar qual foi a 
cidade que esteve no pior cenário em relação à COVID-19, de acordo com o seu número de habitantes. E, consequentemente, qual a cidade teve o melhor comportamento frente à pandemia.

Sendo assim, dividiu-se o número de população pelo número de óbitos de cada cidade e pelos valores obtidos por essa divisão calculou-se a média, o desvio-padrão, a variância e a curtose da amostra utilizando o software Maple 17. A figura 3 apresenta os respectivos valores obtidos.

Figura 3. Média, Desvio-Padrão, Variância e Curtose

\begin{tabular}{r|l}
$\mathrm{mu}:=\operatorname{Mean}(L)$ & $\mu:=0.000768026850023683$ \\
\hline sigma $:=\operatorname{StandardDeviation}(L)$ & $\sigma:=0.000490431346160486$ \\
\hline Variance $(L)$ & $2.4052290529678710^{-7}$ \\
\hline Kurtosis(Normal(mu, sigma) $)$ & 3.000000000
\end{tabular}

Fonte: Autores (2021)

Conforme pode-se observar através dos valores apresentados na figura 3, de um modo geral, as cidades mineiras tiveram um comportamento muito semelhante frente à pandemia, pois considerando número de óbitos e população estimada a variação nos resultados é muito pequena.

Dos 68 municípios do estado de Minas Gerais que não obtiveram óbitos, a tabela 1 apresenta as 8 cidades com maior número populacional.

Tabela1. Cidades mineiras com 0 óbitos

\begin{tabular}{l|r|r} 
Município & Óbitos & População Estimada 2020 \\
\hline Berilo & 0 & 11872 \\
\hline Bonito de Minas & 0 & 11369 \\
\hline São Thomé das Letras & 0 & 10960 \\
\hline Francisco Badaró & 0 & 10321 \\
\hline São Sebastião do Maranhão & 0 & 9963 \\
\hline Paula Cândido & 0 & 9584 \\
\hline Carbonita & 0 & 9414 \\
\hline Angelândia & 0 & 8557
\end{tabular}

Fonte: Autores (2021) 
Conforme pode-se observar tabela 1 , das cidades que tiveram 0 óbitos, a que esteve no melhor cenário foi a cidade de Berilo, considerando sua população estimada de 11.872 habitantes.

Dos 93 municípios do estado de Minas Gerais que obtiveram apenas 1 óbito, a tabela 2 apresenta as 8 cidades com maior número populacional.

Tabela2. Cidades mineiras com apenas 1 óbito

\begin{tabular}{l|r|r|} 
Municipio & Óbitos & População Estimada 2020 \\
\hline Chapada do Norte & 1 & 15345 \\
\hline Chapada Gaúcha & 1 & 13953 \\
\hline Itaipé & 1 & 12836 \\
\hline Ubai & 1 & 12599 \\
\hline Icarai de Minas & 1 & 12097 \\
\hline Guarani & 1 & 8918 \\
\hline Virgínia & 1 & 8663 \\
\hline Ibiai & 1 & 8437
\end{tabular}

Fonte: Autores (2021)

Conforme pode-se observar tabela 2, das cidades que tiveram apenas 1 óbito, a que esteve no melhor cenário foi a cidade de Chapada do Norte, considerando sua população estimada de 15.345 habitantes.

A tabela 3 apresenta as 8 cidades com o pior cenário frente à pandemia da COVID-19 no estado de Minas Gerais considerando o número populacional.

Tabela3. Cidades com o pior cenário frente à pandemia

\begin{tabular}{|l|r|r|r|}
\hline Município & Óbitos & População Estimada 2020 & Análise \\
\hline Aracitaba & 7 & 2059 & $0,34 \%$ \\
\hline Toledo & 17 & 6297 & $0,27 \%$ \\
\hline Jaguaraçu & 8 & 3142 & $0,25 \%$ \\
\hline Santo Antônio Do Aventureiro & 9 & 3602 & $0,25 \%$ \\
\hline Ubaporanga & 31 & 12493 & $0,25 \%$ \\
\hline Araporã & 17 & 6931 & $0,25 \%$ \\
\hline Itapeva & 24 & 9881 & $0,24 \%$ \\
\hline Coromandel & 66 & 27966 & $0,24 \%$ \\
\hline
\end{tabular}

Fonte: Autores (2021)

A cidade de Coromandel registrou 66 óbitos e possui 27.966 habitantes e a cidade de Aracitaba registrou 7 óbitos e possui apenas 2.059 habitantes. 
A tabela 4, que segue abaixo, apresenta as cidades com o melhor e o pior cenário frente à pandemia da COVID-19 no estado de Minas Geraisconsiderando o número populacional.

Tabela 4. Cidades com o melhor e o pior cenário frente à pandemia

\begin{tabular}{|l|r|r|r|}
\hline Municipio & Óbitos & População Estimada 2020 & Análise \\
\hline Berilo & 0 & 11872 & $0,00 \%$ \\
\hline Aracitaba & 7 & 2059 & $0,34 \%$
\end{tabular}

Fonte: Autores (2021)

Conforme pode-se observar na tabela 4 , a cidade de Berilo não registrou nenhum óbito e possui 11.872 habitantes, enquanto a cidade de Aracitaba registrou 7 óbitos e possui apenas 2.059 habitantes. Neste sentido, pode-se afirmar que, no período estudado e relacionando o número de óbitos com o número populacional das cidades, Berilo obteve o melhor cenário frente à pandemia da COVID-19 no estado de Minas Gerais, enquanto Aracitaba obteve o pior cenário.

\section{CONCLUSÃO}

A COVID-19 é uma doençainfecciosaque apresenta uma contaminação agravante, podendo afetar a população de forma significativa e ocasionar um elevado índice de óbitos, por isso, atualmente, o mundo encontra-se em um cenário pandêmico.

Neste sentido, o presente artigo foi desenvolvido com o objetivo de analisar o comportamento da pandemia daCOVID-19 no estado de Minas Geraisdesde o início da pandemia no Brasil, mês de março de 2020, até o dia 17/01/2021, data em que se iniciou a vacinação nesse estado brasileiro.

Para iniciar o estudo, fez-se necessárioextrair bases de dados encontradas no site da Secretaria de Estado de Saúde de Minas Gerais e no site do IBGE, as quais traziam informações como nome dos municípios mineiros, número de óbitos, data dos óbitos e população estimada de cada cidade.

Através da ferramenta Microsoft Excel 2016organizou-se os dados acima mencionados e dividiu-se o número de óbitos pelo número populacional de cada cidade com o intuito de identificar qual foi o percentual de óbitos em relação a população dos munícipios. Após essa etapa, realizou-se cálculos estatísticos da amostra: média, desviopadrão, variância e curtose, utilizando o software Maple 17. 
Após o desenvolvimento dos cálculos, foram realizadasanálises para identificar qual o cenário do estado de Minas Geraisa respeito da COVID-19 no período de estudo.Identificou-se que a cidade de Belo Horizonte teve o maior número de óbitospor apresentar o maior número populacional do estado.Também, identificou-se que a cidade de Aracitaba teve o pior cenário, considerando a população estimada.Ainda,identificouseas cidades que apresentaram 0 óbitos,destacando a localidade de Berilo que apresentou o melhor cenário e, também, relatou-se os municípios que apresentaram apenas 1 óbito, sobressaindo a cidade de Chapada do Norte.

Neste sentido, conclui-se queapós relacionar o número de óbitos com a população estimadados municípios mineiros, o pior cenário frente à pandemia da COVID-19 no estado de Minas Gerais foi da cidade deAracitaba,com7 óbitos do total de 2.059 habitantes. Enquanto a cidade que apresentou o melhor cenário foi Berilo,com 0 óbitos e uma população de 11.872 habitantes. Além disso, pode-se observar que as cidades mineiras, de um modo geral, tiveram um comportamento muito semelhante frente à pandemia, pois a variação nos valores de média, desvio-padrão, variância e curtose obtida foi muito pequena. 


\section{REFERÊNCIAS BIBLIOGRÁFICAS}

A ferramenta essencial para matemática. Maplesoft, 2021. Disponível em: $<$ https://www.maplesoft.com/products/maple/>. Acesso em: 09 abr. 2021.

Análise de Dados com Excel Para Leigos. [S. 1.]: Alta Books, 2020. 344 p.

Disponível em: $\quad<$ https://books.google.com.br/books?id=albdDwAAQBAJ\&hl=ptBR\&source $=$ gbs_navlinks_s $>$. Acesso em: 24 fev. 2021.

Cidades e Estados. IBGE, 2020. Disponível em: <https://www.ibge.gov.br/cidades-eestados/mg.html>. Acesso em: 11 mar. 2021.

Coronavírus Brasil. DATASUS, 2021. Disponível em: <https://covid.saude.gov.br/>. Acesso em: 19 mai. 2021.

Coronavírus SUS. DATASUS, 2021. Disponível em: <https://coronavirusapp.saude.gov.br/app/inicio>. Acesso em: 19 mai. 2021.

Distribuição dos casos de covid-19. Secretaria de Estado de Saúde de Minas Gerais, 2020. Disponível em: <http://coronavirus.saude.mg.gov.br/painel>. Acesso em: 11 mar. 2021.

Manual de Análise de Dados: Estatística e Modelagem Multivariada com Excel®, SPSS ${ }^{\circledR}$ e Stata ${ }^{\circledR}$. [S. 1.]: Elsevier, 2017. 1196 p. Disponível em: $<$ https://books.google.com.br/books?id=SmlaDwAAQBAJ\&printsec=frontcover\&hl=pt $-\mathrm{BR} \# \mathrm{v}=$ onepage \&q\&f$=$ false $>$. Acesso em: 25 fev. 2021.

Microsoft Excel, 2020. Disponível em: <https://www.microsoft.com/pt-br/microsoft365/excel>. Acesso em: 09 abr. 2021. 